\title{
Cardiogenic Shock as consequence of chemotherapy-related dilated cardiomyopathy: a case report
}

\begin{abstract}
The progress in cancer therapy in recent decades has increased the life expectancy of breast cancer, concomitantly with increased morbidity. Exposure to the side effects of chemotherapy may be catastrophic in some cases, especially when it comes to cardiotoxicity, represented by chemotherapy-related dilated cardiomyopathy. The combination of trastuzumab and anthracycline is highly effective in combating primary breast cancer; however, it is associated with a higher incidence of cardiotoxicity when compared to the use of trastuzumab alone. The main consequences are the interruption of the cancer treatment and the frequent cardiac decompensation's, which can even come to cardiogenic shock in its most severe form. Several papers aim to define primary prophylaxis drug measures, but still lack universal consensus in the literature.
\end{abstract}

Keywords: trastuzumab, cardiotoxicity, chemotherapy, dilated cardiomyopathy
Volume 7 Issue I - 2019

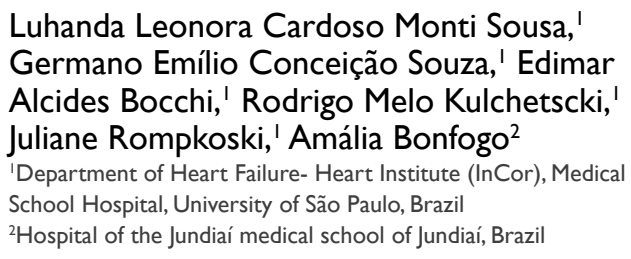

Correspondence: Luhanda Leonora Cardoso Monti Sousa, M.D, Cardiology student in InCor-HCFMUSP, Rua Oscar Freire, 2040 cep: 054090 I I São Paulo-SP/Brazil, Email luhanda_med@hotmail.com

Received: January 27, 2019 | Published: February 13, 2019

\section{Introduction}

Neoplasms and cardiovascular diseases are the main causes of death in the general population. In recent years, the survival of cancer patients has improved exponentially due to several factors, such as early cancer diagnosis, better control of risk factors and advances in oncology therapy, especially chemotherapy. By 2020, the United States estimative is that the number of survivors with cancer reaches its 18 million people. In this scenario, the paradox is established: increased longevity $\mathrm{x}$ greater morbidity. ${ }^{1} \mathrm{~A}$ unique entity called chemotherapyrelated dilated cardiomyopathy (CRDCM), which accounts for about $1 \%$ of all dilated cardiomyopathies, is the cardiotoxic manifestation associated with agents such as anthracyclines (ATC) and trastuzumab. ${ }^{2-4}$ In its most severe form of presentation, it has heart failure with reduced left ventricular ejection fraction (LVEF). ${ }^{1,5}$ Clinically significant CRDCM was defined as a LVEF reduction greater than $10 \%$ for a final value of less than $50 \%$. Decreases in LVEF that do not result in a final ejection fraction below $50 \%$ are generally not classified as clinically significant, although several studies suggest that early subclinical declines in LVEF may have a higher predictive value than originally defined, identified through myocardial injury markers and some indirect echocardiographic signs (echo). ${ }^{1-4,6}$ The combination of ATC with trastuzumab, however, although highly effective against cancer, potentiates the occurrence of cardiotoxicity. ${ }^{4,6-8}$

Cardiotoxic chemotherapy are classified into type I agents whose prototype of are the ATC and determine irreversible lesions. Type II agents, represented by trastuzumab, cause reversible and better prognostic lesions. Grade I cardiotoxicity: asymptomatic LVEF reduction between $10 \%$ and $20 \%$, Grade II: reduction of LVEF below $20 \%$ or below normal and Grade III: HF with reduced and symptomatic LVEF. It can also be classified as acute, subacute and chronic. Acute or subacute cardiotoxicity may occur from the onset until 14 days after the end of chemo and is characterized primarily by cardiac arrhythmias. Chronic cardiotoxicity, represented by CRDCM, has two subtypes according to the onset of symptoms. The first subtype occurs within the first year after the end of chemotherapy, and the second usually after one year after its completion. The main consequence of heart disease in this context are the various episodes of decompensated HF and interruption of chemotherapy. ${ }^{3-6} \mathrm{~A}$ fatal outcome with cardiogenic shock and cardiovascular death is likely to occur. ${ }^{3-6}$

Screening measures aimed at identifying patients at greater risk of developing cardiotoxicity are recommended by cardiology and oncology guidelines, however, so far no medication has been able to prevent the occurrence of CRDCM. ${ }^{5,9,10}$ Once the CRDCM is installed, the treatment does not differ from the conventional therapy for HF of other etiologies..$^{5,9,11-13}$ The present study aims to report a case of CRDCM with biventricular dysfunction and cardiogenic shock that evolved with reverse remodeling after 6 months of drug therapy optimized for HF with reduced LVEF.

\section{Case report}

K.B.S.S, female, 38 years old and antecedent of right breast neoplasm, submitted to total mastectomy and chemotherapy with ATC and transtuzumab for 1 year. After the first 6 months of the end of the chemotherapy regimen, she developed dyspnea on moderate exertion, lower limb edema and nocturnal paroxysmal dyspnea. She went to our institution's ER because of worsening of those symptoms. At admission, she was in regular general condition, preserved peripheral perfusion, jugular turgidity, heart sounds, regular heart rhythm, no murmurs, with B3, crackles in bases, hepatomegaly, without lower limb edema, blood pressure 90x60 $\mathrm{mmHg}$, heart rate of $100 \mathrm{bpm}$. LVEF at the pre treatment was $63 \%$. We performed an echocardiogram that demonstrated biventricular dysfunction with severe LV systolic dysfunction (LVEF 27\%) and grade II diastolic dysfunction, moderate right ventricular (RV) systolic dysfunction without anatomically 
important valvulopathy. She was admitted with a diagnosis of decompensated heart failure. During her stay, a cardiac magnetic resonance imaging (CMR) with functional study was performed and confirmed the biventricular dysfunction seen in echocardiogram, and also diffuse myocardial edema, dilation of the inferior vena cava and hepatic, moderate pericardial effusion, thrombus at the apex of LV and $\mathrm{RV}$, and absence of fibrosis. Angiotensin II receptor blocker (BRA) was started. After about 1 week, the patient evolved with cardiogenic shock, being refer to Coronary Unit and handled with iv dobutamine at a maximum dose of $20 \mathrm{mcg} / \mathrm{kg} / \mathrm{min}$, weaning the inotropic with iv vasodilators and subsequently with captopril in 15 days. In the course of hospitalization, furosemide $40 \mathrm{mg}$ bid, spironolactone $50 \mathrm{mg} \mathrm{qd}$, losartan 50mg qd, bisoprolol $10 \mathrm{mg}$ qd, hydrochlorothiazide $25 \mathrm{mg}$ qd, warfarin $5 \mathrm{mg}$ qd and atorvastatin $20 \mathrm{mg}$ qd were prescribed and gradually optimized. She was then discharged in good health condition. After 6 months of drug therapy for HF, he performed a new CMR that showed a LVEF of 56\%; FEVD 39\% (lower limit of normality) and absence of thrombus. He was followed up in outpatient care at our institution for 6 years, and the drugs were adjusted and optimized. Currently in NYHA functional class I. She was on bisoprolol $15 \mathrm{mg}$ qd and losartan 50mg qd. CMR and Echo were normal (Figure 1 \& 2).

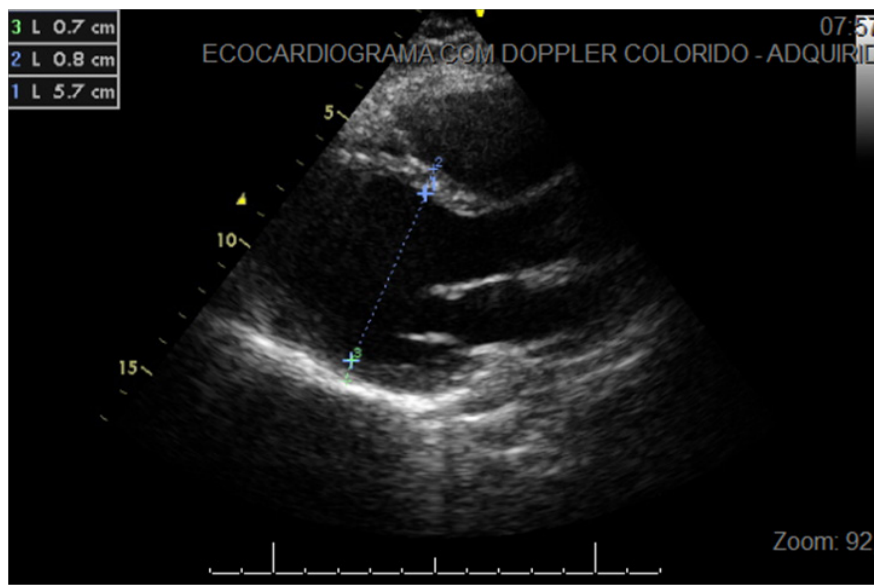

Figure I Echocardiogram (Before)

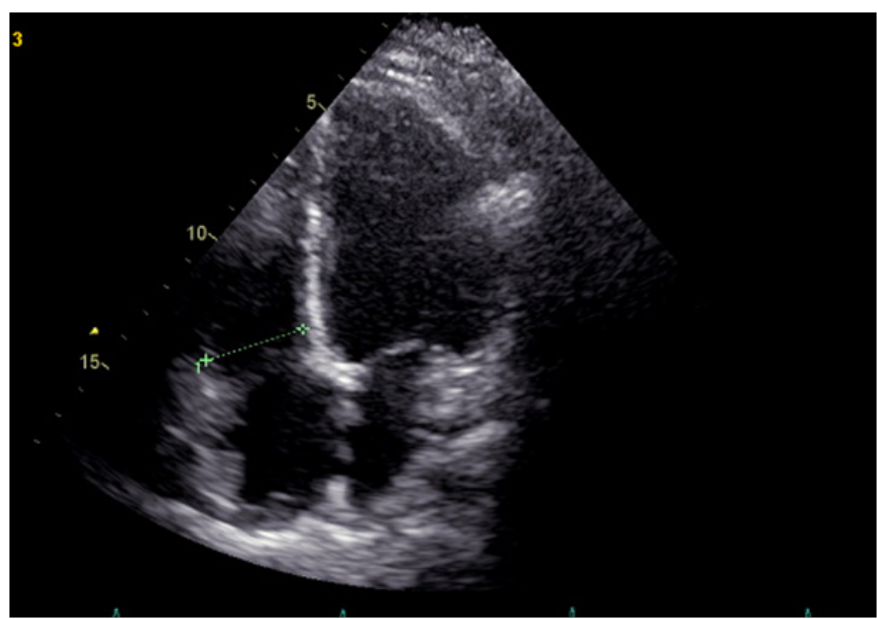

Figure 2 Echocardiogram (After).

\section{Discussion}

The present report reflects the reality of a patient who has gone through all the consequences of the current oncologic cardiology panorama. The life expectancy of breast cancer has increased dramatically in the last decades, which represents the early diagnosis and the new arsenal of available chemotherapy, however patients began to experience the concomitant increase in the side effects of these drugs, such as cardiotoxicity. ${ }^{3,10,14-16}$ The CRDCM courses with reduced FEVE in the majority of cases, which negatively impacts the outcome of these patients, as leads to the interruption of chemo and maybe compromise the cure or the adequate control of the cancer. Our patient had what we call chronic cardiotoxicity and therefore did not need to interrupt the chemotherapy. ${ }^{1,5,16}$ Trastuzumab, approved for treatment of early and metastatic HER2-positive breast cancer, demonstrated a significant benefit in outcomes after 1 year of administrion. ${ }^{1,7}$ The association of Trastuzumab with ATC is widely used and highly effective. However, this combination is related to an increased incidence of cardiotoxicity, reaching $34 \%$, compared to the use of trastuzumab alone, which has an incidence of $0.6 \%$ to $4.5 \% .^{1-4}$

Trastuzumab is a humanized monoclonal antibody that binds to the overexpressed HER-2 protein domain in about $20 \%$ to $25 \%$ of breast cancers. The pathogenesis of trastuzumab-mediated CRDCM has not been fully elucidated, but appears to be linked to HER2 receptor blockade, an important cardioprotector in normal myocytes, which modifies contractile and mitochondrial proteins at structural and functional level, but rarely leads to cell death, explaining the potential for reversibility with drug withdrawal. ${ }^{1-3}$ It starts at the end of the treatment, regardless of dose. ${ }^{5,1,2}$

ATC, CRDCM prototype, damages the myocardium through oxidative stress, resulting in myocardial necrosis and irreversible lesions dose and time-independent, in addition to leaving myocytes susceptible to injury by oxygen reactive species. ${ }^{2,5}$ In the case of adjuvant therapy for primary breast cancer, the use of ATC derivatives caused a reduction of $10 \%$ in LVEF in 10 to $50 \%$ of treated patients, even with doses considered safe for treatment, after 10 to 20 years of treatment. ${ }^{17}$ Our patient made use of this association, demonstrating that summation synergism is actually harmful to the myocardium. Several studies have described a number of potential risk factors, although only age and concomitant therapy actually correlate with increased risk of cardiotoxicity. ${ }^{5,16,17}$

The clinical picture is compatible with decompensated heart failure, and although not described in the literature, cadiogenic shock (CS) is a possible complication. ${ }^{6,9,15}$ Chronic heart failure with reduced $\mathrm{EF}$, independent of etiology, when decompensated, accounts for up to $30 \%$ of cases. Even with all therapeutic advances developed in recent years. In the context of acute coronary syndrome, CS mortality is high, with $80 \%$ in the pre-angioplasty era and $40-60 \%$ in the contemporary era. To date there are no data regarding the actual incidence and prevalence of CS in the population with CRDCM. ${ }^{9}$ The treatment consists of standard CS therapy, with inotropes, vasopressors and mechanical circulatory assistance devices. ${ }^{6,9,15}$ The pharmacological treatment of CRDCM includes the same pharmacological regimens recommended for HF of other etiologies. ${ }^{5,15}$ The first line drugs are: angiotensin converting enzyme inhibitors (ACEI), AT2 receptor blockers (ARBs), beta-blockers (B-blockers), and aldosterone antagonists. ${ }^{15,16,18}$ Recently, the combination of the neprilysin and valsartan inhibitor, recognized by the PARADIGM-HF study, is indicated for HF LVEF $<35 \%$, CF NYHA II-IV, which remain symptomatic despite standard therapy optimized for HF. ${ }^{19}$

It is known that the late recognition of CRDCM is associated with a worse prognosis, including lack of clinical response to pharmacological therapy and terminal HF, as occurred with the present report that was already diagnosed in NYHA functional class III, progressing rapidly for cardiogenic shock. ${ }^{12,16,20,21}$ The 
primary objective of both cardiologists and oncologists has been the early stratification of the risk of cardiotoxicity, since it allows the implementation of therapeutic strategies and the monitoring of progression of cardiac injury and fatal outcomes. ${ }^{15,16}$ However, the development of strategies for monitoring ATC-induced cardiac injury has been challenging. ${ }^{22,23}$ Endomyocardial biopsy is the gold standard, but because it is an invasive and operator-dependent procedure, it is not routinely used. ${ }^{21}$ Measurement of cardiospecific biomarkers, such as cardiac troponin I (cTnI), is a useful diagnostic and prognostic tool. Its increase after the onset of chemo is a marker of risk for future cardiac events, and elevation of cTnI after 1 month of chemotherapy was related to a higher incidence of events when compared to a transient increase. cTnI has a high negative predictive value, allowing the exclusion of long-term monitoring with expensive methods such as echocardiography and cardiac magnetic resonance. ${ }^{13,20,21}$

The use of medications in the prophylaxis of cardiotoxicity mediated by ATC and trastuzumab is a very controversial topic. More recently, the PRADA and MANTICORE trials have shown an impact on CRDCM drug therapy in patients with breast cancer and chemo. ${ }^{8,18}$ Both studies evaluated the prophylaxis of cardiotoxicity caused by chemotherapy with anthracyclines and trastuzumab specifically in early-stage breast cancer. In summary, MANTICORE concludes that perindopril and bisoprolol attenuated the LVEF associated with trastuzumab but did not prevent the primary outcome (LV remodeling) ${ }^{8}$ The PRADA study, however, concluded that in patients with early-stage breast cancer treated with ATC, metoprolol did not affect the overall decline in LVEF, but candesartan provided protection against early LVEF decline. ${ }^{18}$ In 2018, another randomized study evaluating the prevention of $>10 \%$ reduction in LVEF at 6 months and the values of markers of myocardial injury (cTnI, BNP and diastolic dysfunction), concluded that the use of carvedilol significantly reduced myocardial injury evaluated by troponin I levels, as well as the onset of diastolic dysfunction. However, this reduction had no impact on myocardial dysfunction related to cardiotoxicity or the onset of $\mathrm{HF}^{20}$

Prevention is based on the recognition of risk factors, especially the cumulative dose of anthracycline above $550 \mathrm{mg} / \mathrm{m} 2$, and the combination with trastuzumab. ${ }^{16,17,23}$ Most recent guidelines of the American Society of Clinical Oncology and the European Society of Cardiology do not recommend ACE inhibitors or beta-blockers as preventive strategies for potentially cardiotoxic patients in chemo due to limitations in studies (size of sample and measures of effectiveness). ${ }^{15,16}$ No studies have reported the long-term outcome of prophylactic therapies after cessation of both anthracyclines and cardio protective therapies. Therefore, to date, the data are not sufficient to support the routine use of primary prevention in this scenario, requiring larger studies with long-term follow-up and in patients at higher risk. ${ }^{5,15,16}$

\section{Conclusion}

The present report aimed to demonstrate the case of a patient with late diagnosis (after having completed chemotherapy for breast cancer with Trastuzumab and ACT), CRDCM and biventricular dysfunction, who presented cardiogenic shock, a rare entity in this context and high mortality, but progressed with complete reverse remodeling after drug therapy optimized for HF. Studies are required to better understand the role of primary prophylaxis of cardiotoxicity in these patients, in order to avoid such catastrophic and potentially fatal presentation.

\section{Acknowledgments}

None.

\section{Conflicts of interest}

Authors declare that there is no conflict of interest.

\section{References}

1. Mukku RB, Fonarow GC, Watson KE, et al. Heart Failure Therapies for End-Stage Chemotherapy-Induced Cardiomyopathy. J Card Fail. 2016;22(6):439-448.

2. Fanous I, Dillon P. Cancer treatment-related cardiac toxicity: prevention, assessment and management. Med Oncol. 2016;33(8):84.

3. Ewer MS, Vooletich MT, Durand JB, et al. Reversibility of trastuzumabrelated cardiotoxicity: new insights based on clinical course and response to medical treatment. Clin Oncol. 2005;23(31):7820-7826.

4. Keefe DL. Trastuzumab-associated cardiotoxicity. Cancer. 2002;95(7):1592-1600

5. Kalil Filho R, Hajjar LA, Bacal F, et al. I Diretriz Brasileira de CardioOncologia da Sociedade Brasileira de Cardiologia. Arq Bras Cardiol. 2011;96(2 Suppl1):1-52.

6. Telli ML, Hunt SA, Carlson RW, et al. Trastuzumab-related cardiotoxicity: calling into question the concept of reversibility. $J$ Clin Oncol. 2007;25(23):3525-3533.

7. Meattini I, Curigliano G, Terziani F, et al. SAFE trial: an ongoing randomized clinical study to assess the role of cardiotoxicity prevention in breast cancer patients treated with anthracyclines with or without trastuzumab. Med Oncol. 2017;34(5):75.

8. Pituskin E, Haykowsky M, Mackey JR, et al. Rationale and design of the Multidisciplinary Approach to Novel Therapies in Cardiology Oncology Research Trial (MANTICORE 101--Breast): a randomized, placebo-controlled trial to determine if conventional heart failure pharmacotherapy can prevent trastuzumab-mediated left ventricular remodeling among patients with HER2+ early breast cancer using cardiac MRI. BMC cancer. 2011;11:318.

9. Diepen SV, Katz JN, Albert NM, et al. Contemporary Management of Cardiogenic Shock: A Scientific Statement from the American Heart Association. Circulation. 2017;136(16):e232-e268.

10. Minichillo S, Gallelli I, Cubelli MI, et al. Trastuzumab resumption after extremely severe cardiotoxicity in metastatic breast cancer patient: a case report. BMC Cancer. 2017;17(1):722.

11. Martín M, Esteva FJ, Alba E, et al. Minimizing cardiotoxicity while optimizing treatment efficacy with trastuzumab: review and expert recommendations. Oncologist. 2009;14(1):1-11.

12. Gujral DM, Lloyd G, Bhattacharyya S. Effect of prophylactic betablocker or ACE inhibitor on cardiac dysfunction \& heart failure during anthracycline chemotherapy \pm trastuzumab. Breast. 2018;37:64-71.

13. Cardinale D, Colombo A, Torrisi R, et al. Trastuzumab-induced cardiotoxicity: clinical and prognostic implications of troponin I evaluation. J Clin Oncol. 2010;28(25):3910-3916.

14. Huzno J, Les D, Sarzyczny-Slota D, et al. Cardiac side effects of trastuzumab in breast cancer patients-single centers experiences. Wspolczesna Onkol. 2013;17(2):190-195.

15. Rohde LEPR, Montera MW, Bocchi, EA, et al. Diretriz Brasileira de Insuficiência Cardíaca Crônica e Aguda. Arq Bras Cardiol. 2018;111(3):436-539. 
16. Zamorano JL, Lancellotti P, Rodriguez Muñoz D, et al. 2016 ESC Position Paper on cancer treatments and cardiovascular toxicity developed under the auspices of the ESC Committee for Practice Guidelines: The Task Force for cancer treatments and cardiovascular toxicity of the European Society of Cardiology (ESC). Eur Heart J. 2016;37(36):2768-2801.

17. Perez EA, Suman VJ, Davidson NE, et al. Effect of doxorubicin plus cyclophosphamide on left ventricular ejection fraction in patients with breast cancer in the North Central Cancer Treatment Group N9831 Intergroup Adjuvant Trial. J Clin Oncol. 2004;22(18):3700-3704.

18. Gulati G, Heck SL, Ree AH, et al. Prevention of cardiac dysfunction during adjuvant breast cancer therapy (PRADA): a $2 \times 2$ factorial, randomized, placebo-controlled, double-blind clinical trial of candesartan and metoprolol. Eur Heart J. 2016;37(21):1671-1680.

19. McMurray JJ, Packer M, DesaiAS, et al. Angiotensin-neprilysin inhibition versus enalapril in heart failure. $N$ Engl J Med. 2014;371(11):993-1004
20. Avila MS, Ayub-Ferreira SM, de Barros Wanderley MR Jr, et al. Carvedilol for Prevention of Chemotherapy-Related Cardiotoxicity: The CECCY Trial. J Am Coll Cardiol. 2018;71(20):2281-2290.

21. Armenian, SH, Lacchetti C, Barac A, et al. Prevention and monitoring of cardiac dysfunction in survivors of adult cancers: american society of clinical Oncology clinical practice guideline. J Clin Oncol. 2017;35(8):893-911.

22. O'Brien, PJ. Cardiac troponin is the most effective translational safety biomarker for myocardial injury in cardiotoxicity. Toxicology. 2008;245(3):206-218.

23. Ng R, Better N, Green MD. Anticancer agents and cardiotoxicity. Semin Oncol. 2006;33(1):2-14. 